\title{
A second transition state for chain transfer to monomer in olefin polymerization promoted by Group 4 metal catalysts
}

Giovanni Talarico ${ }^{\ddagger 1}$ and Peter H.M. Budzelaar ${ }^{\dagger}$

Dipartimento di Chimica, Università di Napoli, Via Cintia, 80126 Napoli, Italy, and Department of Chemistry,

University of Manitoba, Winnipeg MB R3T 2N2, Canada

\section{Supporting Information}

\section{Details of calculations}

\section{General}

All structures were fully optimized, without any constraints. All stationary points were characterized by a vibrational analysis, and thermal corrections (ZPE, enthalpy, entropy; 273K, 1 bar) were calculated from these using standard formulae of statistical thermodynamics (imaginary frequencies were excluded).

\section{Path $A$ and $C$ transition states for systems 1-6 ('Bu to propene transfer)}

These calculations (Table S1) were performed with Gaussian03, ${ }^{1}$ using the optimizer contained in that program. They all used the B3LYP functional ${ }^{2}$ and the SVP basis set on the light atoms, ${ }^{3}$ LANL2DZ basis and ECP on the metal atoms. ${ }^{4}$

\section{Path $A$ and $C$ transition states and second-order saddle points for systems 1 ('Bu/propene, Et/ethene and ${ }^{n}$ Pr/ethene transfer)}

These calculations (Tables S2 and S3) were performed with Gaussian98 ${ }^{5}$ and Turbomole ${ }^{6}$ in combination with external optimizers. For saddle points, we used the OPTIMIZE routine of Baker and co-workers; ${ }^{7}$ second-order saddle points were located using a development version of our own external optimizer, which is based on the same general principles but has some additional options. ${ }^{8}$ Gaussian calculations were performed with the B3LYP functional, ${ }^{2}$ the SVP basis set on the light atoms, ${ }^{3}$ LANL2DZ basis and ECP on the metal atoms. ${ }^{4}$ Turbomole optimizations were carried out using the b3-lyp functional ${ }^{2}$ and the SV(P) basis set ${ }^{3}$ (small-core pseudopotential on $\mathrm{Zr}$ and $\mathrm{Hf}){ }^{9}{ }^{9}$ improved single-point energies were then calculated at the b3-lyp level using the TZVPP basis set. $^{10}$

\footnotetext{
† Università di Napoli

1 To whom correspondence should be addressed. E-mail: talarico@unina.it

${ }^{\dagger}$ University of Manitoba
} 
Table S1. Total (a.u.) and relative (kcal/mol) energies for systems 1-6 calculated with Gaussian03 (B3LYP functional).

\begin{tabular}{|c|c|c|c|c|c|c|}
\hline \multirow[t]{2}{*}{ System } & \multirow[t]{2}{*}{ Point } & \multirow{2}{*}{$\begin{array}{r}E \\
\text { B3LYP/SVP }\end{array}$} & \multirow{2}{*}{$\begin{array}{r}E+\text { ZPE } \\
\text { B3LYP/SVP }\end{array}$} & \multicolumn{2}{|l|}{$G$} & \multirow[b]{2}{*}{$G_{\text {rel }}$} \\
\hline & & & & B3LYP/SVP & $E_{\text {rel }}$ & \\
\hline \multirow[t]{2}{*}{$1-\mathrm{Ti}$} & $\mathrm{TS}(\mathrm{C})$ & -720.208708 & -719.841300 & -719.886851 & 0.0 & 0.0 \\
\hline & TS(A) & -720.209927 & -719.840900 & -719.885903 & -0.8 & 0.6 \\
\hline \multirow[t]{2}{*}{$1-Z r$} & $\mathrm{TS}(\mathrm{C})$ & -708.735600 & -708.370142 & -708.417786 & 0.0 & 0.0 \\
\hline & TS(A) & -708.749389 & -708.382179 & -708.428836 & -8.7 & -6.9 \\
\hline \multirow[t]{2}{*}{ 1-Hf } & $\mathrm{TS}(\mathrm{C})$ & -711.073094 & -710.707026 & -710.754741 & 0.0 & 0.0 \\
\hline & $\mathrm{TS}(\mathrm{A})$ & -711.074786 & -710.707272 & -710.754152 & -1.1 & 0.4 \\
\hline \multirow[t]{2}{*}{$2-\mathrm{Ti}$} & $\mathrm{TS}(\mathrm{C})$ & -1113.076103 & -1112.433164 & -1112.494700 & 0.0 & 0.0 \\
\hline & TS(A) & -1113.063338 & -1112.418607 & -1112.478201 & 8.0 & 10.4 \\
\hline \multirow[t]{2}{*}{$2-\mathrm{Zr}$} & $\mathrm{TS}(\mathrm{C})$ & -1101.611548 & -1100.969943 & -1101.032747 & 0.0 & 0.0 \\
\hline & $\mathrm{TS}(\mathrm{A})$ & -1101.611435 & -1100.968262 & -1101.029240 & 0.1 & 2.2 \\
\hline \multirow[t]{2}{*}{ 2-Hf } & $\mathrm{TS}(\mathrm{C})$ & -1103.945098 & -1103.302895 & -1103.365883 & 0.0 & 0.0 \\
\hline & TS(A) & -1103.933919 & -1103.290778 & -1103.353012 & 7.0 & 8.1 \\
\hline \multirow[t]{2}{*}{ 3-Ti } & $\mathrm{TS}(\mathrm{C})$ & -1088.237261 & -1087.816421 & -1087.866906 & 0.0 & 0.0 \\
\hline & TS(A) & -1088.24349 & -1087.821561 & -1087.871242 & -3.9 & -2.7 \\
\hline \multirow[t]{2}{*}{$3-\mathrm{Zr}$} & $\mathrm{TS}(\mathrm{C})$ & -1076.763416 & -1076.344779 & -1076.395881 & 0.0 & 0.0 \\
\hline & $\mathrm{TS}(\mathrm{A})$ & -1076.78139 & -1076.361176 & -1076.412314 & -11.3 & -10.3 \\
\hline \multirow[t]{2}{*}{ 3-Hf } & $\mathrm{TS}(\mathrm{C})$ & -1079.101005 & -1078.681944 & -1078.734625 & 0.0 & 0.0 \\
\hline & TS(A) & -1079.106718 & -1078.686307 & -1078.737740 & -3.6 & -2.0 \\
\hline \multirow[t]{2}{*}{$4-\mathrm{Ti}$} & $\mathrm{TS}(\mathrm{C})$ & -1265.077752 & -1264.495031 & -1264.556783 & 0.0 & 0.0 \\
\hline & $\mathrm{TS}(\mathrm{A})$ & -1265.086169 & -1264.501030 & -1264.560652 & -5.3 & -2.4 \\
\hline \multirow[t]{2}{*}{$4-\mathrm{Zr}$} & $\mathrm{TS}(\mathrm{C})$ & n.d. & n.d & n.d & & \\
\hline & $\mathrm{TS}(\mathrm{A})$ & -1253.603366 & -1253.020762 & -1253.082084 & & \\
\hline \multirow[t]{2}{*}{ 4-Hf } & $\mathrm{TS}(\mathrm{C})$ & -1255.931872 & -1255.350377 & -1255.414076 & 0.0 & 0.0 \\
\hline & $\mathrm{TS}(\mathrm{A})$ & -1255.937317 & -1255.354729 & -1255.416551 & -3.4 & -1.6 \\
\hline \multirow[t]{2}{*}{$5-\mathbf{T i}$} & $\mathrm{TS}(\mathrm{C})$ & -974.401624 & -973.835485 & -973.892644 & 0.0 & 0.0 \\
\hline & $\mathrm{TS}(\mathrm{A})$ & -974.407384 & -973.839595 & -973.895786 & -3.6 & -2.0 \\
\hline \multirow[t]{2}{*}{$5-\mathrm{Zr}$} & $\mathrm{TS}(\mathrm{C})$ & n.d & n.d & n.d & & \\
\hline & TS(A) & -962.927196 & -962.362008 & -962.419334 & & \\
\hline \multirow[t]{2}{*}{ 5-Hf } & $\mathrm{TS}(\mathrm{C})$ & -965.256189 & -964.691477 & -964.751055 & 0.0 & 0.0 \\
\hline & TS(A) & -965.259794 & -964.694487 & -964.752443 & -2.3 & -0.9 \\
\hline \multirow[t]{2}{*}{ 6-Ti } & $\mathrm{TS}(\mathrm{C})$ & -1147.578273 & -1146.915262 & -1146.980236 & 0.0 & $\overline{0.0}$ \\
\hline & $\mathrm{TS}(\mathrm{A})$ & -1147.563824 & -1146.899306 & -1146.963350 & 9.1 & 10.6 \\
\hline \multirow[t]{2}{*}{ 6-Zr } & $\mathrm{TS}(\mathrm{C})$ & -1136.106934 & -1135.445437 & -1135.512424 & 0 & 0 \\
\hline & TS(A) & -1136.094774 & -1135.430298 & -1135.493997 & 7.6 & 11.6 \\
\hline \multirow[t]{2}{*}{ 6-Hf } & $\mathrm{TS}(\mathrm{C})$ & -1138.451879 & -1137.789219 & -1137.855121 & 0.0 & 0.0 \\
\hline & $\mathrm{TS}(\mathrm{A})$ & -1138.425121 & -1137.760748 & -1137.825444 & 16.8 & 18.6 \\
\hline
\end{tabular}


Table S2. Total (a.u.) and relative (kcal/mol) energies for $\mathrm{Cp}_{2} \mathrm{M}$ systems calculated with Turbomole (b3-lyp functional).

\begin{tabular}{|c|c|c|c|c|c|c|c|c|c|c|c|}
\hline \multirow{2}{*}{$\begin{array}{l}\text { Chain/ } \\
\text { olefin }\end{array}$} & \multirow{2}{*}{$\frac{\text { Metal }}{\mathrm{Ti}}$} & \multirow{2}{*}{$\frac{\text { Point }}{\text { TS(C) }}$} & \multicolumn{2}{|l|}{$E$} & $E_{\text {rel }}$ & $G_{\text {rel }}$ & \multicolumn{2}{|c|}{$\begin{array}{c}\text { imags / 2nd } \\
\left(\mathrm{cm}^{-1}\right)^{\mathrm{a}}\end{array}$} & \multirow{2}{*}{$\begin{array}{r}\boldsymbol{E} \\
\text { b3-lyp/tzvpp } \\
-1511.78544\end{array}$} & \multirow{2}{*}{$\begin{array}{r}\boldsymbol{E}_{\text {rel }} \\
1.64\end{array}$} & \multirow{2}{*}{$\frac{\boldsymbol{G}_{\text {rel }}}{0.65}$} \\
\hline & & & -1510.88854 & 0.32803 & 0.44 & 0.00 & I613.4 & 159.8 & & & \\
\hline \multirow[t]{8}{*}{ Propene } & & TS(A) & -1510.88925 & 0.32960 & 0.00 & 0.54 & i1113.8 & 137.6 & -1511.78805 & 0.00 & 0.00 \\
\hline & & $2^{\text {nd }} \mathrm{SP}$ & -1510.87976 & 0.32892 & 5.95 & 6.07 & i1108.4 & i131.1 & -1511.77737 & 6.70 & 6.27 \\
\hline & $\mathrm{Zr}$ & $\mathrm{TS}(\mathrm{C})$ & -708.65346 & 0.32364 & 7.05 & 5.53 & I616.1 & 126.0 & -709.44580 & 8.70 & 7.18 \\
\hline & & TS(A) & -708.66470 & 0.32606 & 0.00 & 0.00 & i1028.4 & 110.7 & -709.45966 & 0.00 & 0.00 \\
\hline & & $2^{\text {nd }} \mathrm{SP}$ & -708.65082 & 0.32408 & 8.71 & 7.46 & $i 993.0$ & $i 96.9$ & -709.44388 & 9.90 & 8.66 \\
\hline & $\mathrm{Hf}$ & $\mathrm{TS}(\mathrm{C})$ & -709.61457 & 0.32305 & 1.19 & 0.02 & i548.5 & 128.2 & -710.40895 & 3.06 & 1.88 \\
\hline & & TS(A) & -709.61648 & 0.32493 & 0.00 & 0.00 & $i 1033.9$ & 109.0 & -710.41382 & 0.00 & 0.00 \\
\hline & & $2^{\text {nd }} \mathrm{SP}$ & -709.60980 & 0.32428 & 4.19 & 3.78 & $i 1087.4$ & $i 116.0$ & -710.40540 & 5.29 & 4.88 \\
\hline Et/ & $\mathrm{Ti}$ & TS(C) & -1393.11978 & 0.24656 & 10.87 & 9.10 & $i 893.2$ & 179.5 & -1393.87401 & 12.85 & 11.08 \\
\hline \multirow[t]{8}{*}{ Ethene } & & TS(A) & -1393.13709 & 0.24938 & 0.00 & 0.00 & i1094.7 & 173.5 & -1393.89449 & 0.00 & 0.00 \\
\hline & & $2^{\text {nd }} \mathrm{SP}$ & -1393.11256 & 0.24813 & 15.39 & 14.61 & i1203.2 & i177.8 & -1393.86811 & 16.55 & 15.77 \\
\hline & $\mathrm{Zr}$ & $\mathrm{TS}(\mathrm{C})$ & -590.88324 & 0.24255 & 15.11 & 12.57 & i861.8 & 135.6 & -591.53323 & 17.22 & 14.67 \\
\hline & & TS(A) & -590.90731 & 0.24660 & 0.00 & 0.00 & i1093.9 & 134.9 & -591.56066 & 0.00 & 0.00 \\
\hline & & $2^{\text {nd }} \mathrm{SP}$ & -590.88194 & 0.24442 & 15.92 & 14.55 & i1069.2 & $i 129.5$ & -591.53260 & 17.61 & 16.24 \\
\hline & $\mathrm{Hf}$ & $\mathrm{TS}(\mathrm{C})$ & -591.84436 & 0.24275 & 8.76 & 6.90 & i788.8 & 140.3 & -592.49650 & 11.09 & 9.22 \\
\hline & & TS(A) & -591.85833 & 0.24572 & 0.00 & 0.00 & i1078.0 & 129.7 & -592.51417 & 0.00 & 0.00 \\
\hline & & $2^{\text {nd }} \mathrm{SP}$ & -591.84199 & 0.24379 & 10.25 & 9.04 & i1059.0 & i133.4 & -592.49526 & 11.87 & 10.66 \\
\hline${ }^{n} \mathrm{Pr} /$ & $\mathrm{Ti}$ & TS(C) & -1432.37910 & 0.27411 & 7.47 & 5.94 & $i 731.2$ & 171.9 & -1433.18070 & 9.08 & 7.55 \\
\hline \multirow[t]{8}{*}{ Ethene } & & TS(A) & -1432.39101 & 0.27655 & 0.00 & 0.00 & i1061.4 & 165.3 & -1433.19517 & 0.00 & 0.00 \\
\hline & & $2^{\text {nd }} \mathrm{SP}$ & -1432.37106 & 0.27493 & 12.52 & 11.50 & i1140.3 & i165.9 & -1433.17371 & 13.47 & 12.45 \\
\hline & $\mathrm{Zr}$ & $\mathrm{TS}(\mathrm{C})$ & -630.14231 & 0.26944 & 12.25 & 10.16 & i761.6 & 129.2 & -630.83957 & 14.22 & 12.13 \\
\hline & & TS(A) & -630.16182 & 0.27277 & 0.00 & 0.00 & i1066.0 & 136.5 & -630.86224 & 0.00 & 0.00 \\
\hline & & $2^{\text {nd }} \mathrm{SP}$ & -630.14065 & 0.27078 & 13.29 & 12.04 & $i 1012.3$ & i116.8 & -630.83850 & 14.90 & 13.65 \\
\hline & $\mathrm{Hf}$ & TS(C) & -631.10337 & 0.26913 & 6.17 & 4.70 & $i 687.9$ & 132.9 & -631.80282 & 8.34 & 6.87 \\
\hline & & TS(A) & -631.11320 & 0.27147 & 0.00 & 0.00 & i1048.0 & 131.9 & -631.81611 & 0.00 & 0.00 \\
\hline & & $2^{\text {nd }} \mathrm{SP}$ & -631.10022 & 0.27054 & 8.14 & 7.56 & i1023.7 & i124.1 & -631.80066 & 9.70 & 9.11 \\
\hline
\end{tabular}

${ }^{a}$ For the regular transition states, the second (real) frequency given is the one that corresponds to "movement" between the path A and C transition states. This is not the lowest real vibration. 
Table S3. Total (a.u.) and relative (kcal/mol) energies for $\mathrm{Cp}_{2} \mathrm{M}$ systems calculated with Gaussian-98 (B3LYP functional).

\begin{tabular}{|c|c|c|c|c|c|c|c|c|}
\hline \multirow{2}{*}{$\begin{array}{l}\text { Chain/ } \\
\text { olefin }\end{array}$} & \multirow{2}{*}{$\frac{\text { Metal }}{\mathrm{Ti}}$} & \multirow{2}{*}{$\frac{\text { Point }}{\text { TS(C) }}$} & \multicolumn{2}{|l|}{$\begin{array}{r}E \\
\text { B3LYP/SVP }\end{array}$} & \multirow{2}{*}{$\begin{array}{r}\frac{\boldsymbol{E}_{\text {rel }}}{} \\
0.76\end{array}$} & \multirow{2}{*}{$\frac{\boldsymbol{G}_{\text {rel }}}{0.00}$} & \multicolumn{2}{|c|}{$\begin{array}{c}\underset{\left(\mathrm{cm}^{-1}\right)^{\mathrm{a}}}{\operatorname{imgs} / \text { 2nd }} \\
\end{array}$} \\
\hline & & & -720.20871 & 0.32730 & & & $i 562.5$ & 160.6 \\
\hline \multirow[t]{8}{*}{ Propene } & & TS(A) & -720.20993 & 0.32939 & 0.00 & 0.55 & i1104.1 & 146.4 \\
\hline & & $2^{\text {nd }} S P$ & -720.19997 & 0.32785 & 6.25 & 5.83 & i1089.3 & i141.4 \\
\hline & $\mathrm{Zr}$ & $\mathrm{TS}(\mathrm{C})$ & -708.73572 & 0.32389 & 8.56 & 7.38 & $i 591.5$ & 127.0 \\
\hline & & $\mathrm{TS}(\mathrm{A})$ & -708.74936 & 0.32577 & 0.00 & 0.00 & i1034.8 & 112.1 \\
\hline & & $2^{\text {nd }} S P$ & -708.73398 & 0.32384 & 9.65 & 8.44 & i820.4 & $i 32.0$ \\
\hline & $\mathrm{Hf}$ & $\mathrm{TS}(\mathrm{C})$ & -711.07318 & 0.32407 & 0.97 & 0.12 & i491.3 & 135.3 \\
\hline & & TS(A) & -711.07472 & 0.32542 & 0.00 & 0.00 & i1012.2 & 112.9 \\
\hline & & $2^{\text {nd }} S P$ & -711.06767 & 0.32501 & 4.42 & 4.16 & i1045.8 & i120.9 \\
\hline Et/ & $\mathrm{Ti}$ & $\mathrm{TS}(\mathrm{C})$ & -602.33849 & 0.24656 & 11.57 & 9.53 & i826.4 & 182.4 \\
\hline \multirow[t]{8}{*}{ Ethene } & & TS(A) & -602.35693 & 0.24982 & 0.00 & 0.00 & i1067.1 & 176.4 \\
\hline & & $2^{\text {nd }} S P$ & -602.33142 & 0.24791 & 16.01 & 14.81 & i1166.0 & i178.0 \\
\hline & $\mathrm{Zr}$ & $\mathrm{TS}(\mathrm{C})$ & -590.86424 & 0.24339 & 16.88 & 15.15 & i842.9 & 141.3 \\
\hline & & TS(A) & -590.89113 & 0.24614 & 0.00 & 0.00 & i1092.6 & 136.2 \\
\hline & & $2^{\text {nd }} S P$ & -590.86340 & 0.24436 & 17.40 & 16.29 & i1013.4 & i112.7 \\
\hline & $\mathrm{Hf}$ & $\mathrm{TS}(\mathrm{C})$ & -593.20227 & 0.24368 & 8.86 & 7.38 & $i 725.4$ & 159.4 \\
\hline & & TS(A) & -593.21639 & 0.24604 & 0.00 & 0.00 & i1038.3 & 137.0 \\
\hline & & $2^{\text {nd }} S P$ & -593.19876 & 0.24448 & 11.06 & 10.08 & i1011.4 & $i 145.6$ \\
\hline${ }^{n} \mathrm{Pr} /$ & $\mathrm{Ti}$ & $\mathrm{TS}(\mathrm{C})$ & -641.63154 & 0.27326 & 8.08 & 5.86 & -660.8 & 172.3 \\
\hline \multirow[t]{8}{*}{ Ethene } & & TS(A) & -641.64442 & 0.27680 & 0.00 & 0.00 & -1060.9 & 167.3 \\
\hline & & $2^{\text {nd }} S P$ & -641.62353 & 0.27423 & 13.11 & 11.50 & -1107.1 & -164.9 \\
\hline & $\mathrm{Zr}$ & $\mathrm{TS}(\mathrm{C})$ & -630.15695 & 0.26969 & 14.03 & 12.21 & -734.0 & 131.9 \\
\hline & & TS(A) & -630.17931 & 0.27260 & 0.00 & 0.00 & -1070.7 & 135.2 \\
\hline & & $2^{\text {nd }} S P$ & -630.15558 & 0.27026 & 14.88 & 13.42 & -966.6 & -110.3 \\
\hline & $\mathrm{Hf}$ & $\mathrm{TS}(\mathrm{C})$ & -632.49484 & 0.27018 & 6.18 & 4.85 & -619.1 & 147.5 \\
\hline & & TS(A) & -632.50470 & 0.27230 & 0.00 & 0.00 & -1018.8 & 137.6 \\
\hline & & $2^{\text {nd }} S P$ & -632.49047 & 0.27071 & 8.93 & 7.93 & -995.2 & -136.9 \\
\hline
\end{tabular}

${ }^{\text {a }}$ For the regular transition states, the second (real) frequency given is the one that corresponds to "movement" between the path $\mathrm{A}$ and $\mathrm{C}$ transition states. This is not the lowest real vibration. 


\section{References}

(1) Gaussian 03, Revision C.02, Frisch, M.J.; Trucks, G.W.; Schlegel, H.B.; Scuseria, G.E.; Robb, M.A.; Cheeseman, J.R.; Montgomery, Jr., J.A.; Vreven, T.; Kudin, K.N.; Burant, J.C.; Millam, J.M.; Iyengar, S.S.; Tomasi, J.; Barone, V.; Mennucci, B.; Cossi, M.; Scalmani, G.; Rega, N.; Petersson, G.A.; Nakatsuji, H.; Hada, M.; Ehara, M.; Toyota, K.; Fukuda, R.; Hasegawa, J.; Ishida, M.; Nakajima, T.; Honda, Y.; Kitao, O.; Nakai, H.; Klene, M.; Li, X.; Knox, J.E.; Hratchian, H.P.; Cross, J. B.; Bakken, V.; Adamo, C.; Jaramillo, J.; Gomperts, R.; Stratmann, R.E.; Yazyev, O.; Austin, A.J.; Cammi, R.; Pomelli, C.; Ochterski, J.W.; Ayala, P.Y.; Morokuma, K.; Voth, G.A.; Salvador, P.; Dannenberg, J.J.; Zakrzewski, V.G.; Dapprich, S.; Daniels, A.D.; Strain, M.C.; Farkas, O.; Malick, D.K.; Rabuck, A.D.; Raghavachari, K.; Foresman, J.B.; Ortiz, J.V.; Cui, Q.; Baboul, A.G.; Clifford, S.; Cioslowski, J.; Stefanov, B.B.; Liu, G.; Liashenko, A.; Piskorz, P.; Komaromi, I.; Martin, R.L.; Fox, D.J.; Keith, T.; Al-Laham, M.A.; Peng, C.Y.; Nanayakkara, A.; Challacombe, M.; Gill, P.M.W.; Johnson, B.; Chen, W.; Wong, M.W.; Gonzalez, C.; and Pople, J.A.; Gaussian, Inc., Wallingford CT, 2004.

(2) (a) Lee, C.; Yang, W.; Parr, R.G. Phys. Rev. B 1988, 37, 785; (b) Becke, A.D. J. Chem. Phys. 1993, 98, 1372; c) Becke, A.D. J. Chem. Phys. 1993, 98, 5648; note that the Turbomole functional "b3-lyp" is not identical to the Gaussian "B3LYP" functional.

(3) Schäfer, A.; Horn, H.; Ahlrichs, R. J. Chem. Phys. 1992, 97, 2571.

(4) (a) Dunning Jr., T.H.; Hay, P.J. in "Modern Theoretical Chemistry", Schaefer III, H.F., Ed., Vol. 3 (Plenum, New York, 1976) 1-28; (b) Hay, P.J.; Wadt, W.R. J. Chem. Phys. 1985, 82, 270; (c) Wadt, W.R.; Hay, P.J. J. Chem. Phys. 1985, 82, 284; (d) Hay, P.J.; Wadt, W.R. J. Chem. Phys. 1985, 82, 299.

(5) Gaussian 98, Revision A.11.4: Frisch, M.J.; Trucks, G.W.; Schlegel, H.B.; Scuseria, G.E.; Robb, M.A.; Cheeseman, J.R.; Zakrzewski, V.G.; Montgomery, Jr., J.A.; Stratmann, R.E.; Burant, J.C.; Dapprich, S.; Millam, J.M.; Daniels, A.D.; Kudin, K.N.; Strain, M.C.; Farkas, O.; Tomasi, J.; Barone, V.; Cossi, M.; Cammi, R.; Mennucci, B.; Pomelli, C.; Adamo, C.; Clifford, S.; Ochterski, J.; Petersson, G.A.; Ayala, P.Y.; Cui, Q.; Morokuma, K.; Rega, N.; Salvador, P.; Dannenberg, J.J.; Malick, D.K.; Rabuck, A.D.; Raghavachari, K.; Foresman, J.B.; Cioslowski, J.; Ortiz, J.V.; Baboul, A.G.; Stefanov, B.B.; Liu, G.; Liashenko, A.; Piskorz, P.; Komaromi, I.; Gomperts, R.; Martin, R.L.; Fox, D.J.; Keith, T.; Al-Laham, M.A.; Peng, C.Y.; Nanayakkara, A.; Challacombe, M.; Gill, P.M.W.; Johnson, B.; Chen, W.; Wong, M.W.; Andres, J.L.; Gonzalez, C.; Head-Gordon, M.; Replogle, E.S.; Pople, J.A. Gaussian, Inc., Pittsburgh PA, 2002.

(6) (a) Ahlrichs, R.; Bär, M.; Häser, M.; Horn, H.; Kölmel, C. Chem. Phys. Lett. 1989, 162, 165; (b) Treutler, O.; Ahlrichs, R. J. Chem. Phys., 1995, 102, 346; (c) Ahlrichs, R.; Bär, M.; Baron, H.-P.; Bauernschmitt, R.; Böcker, S.; Ehrig, M.; Eichkorn, K.; Elliott, S.; Furche, F.; Haase, F.; Häser, M.; Hättig, C.; Horn, H.; Huber, C.; Huniar, U.; Kattannek, M.; Köhn, A.; Kölmel, C.; Kollwitz, M.; May, K.; Ochsenfeld, C.; Öhm, H.; Schäfer, A.; Schneider, U.; Treutler, O.; Tsereteli, K.; Unterreiner, B.; Von Arnim, M.; Weigend, F.; Weis, P.; Weiss H. Turbomole Version 5, January 2002. Theoretical Chemistry Group, University of Karlsruhe.

(7) Baker, J. J. Comput. Chem. 1986, 7, 385; PQS version 2.4, 2001, Parallel Quantum Solutions, Fayetteville, Arkansas, USA; the Baker optimizer is available separately from PQS upon request.

(8) Budzelaar, P.H.M., to be published.

(9) Andrae, D.; Haeussermann, U.; Dolg, M.; Stoll, H.; Preuss, H. Theor. Chim. Acta 1990, 77, 123.

(10) Schäfer, A.; Huber, C. Ahlrichs, R. J. Chem. Phys. 1994, 100, 5829. 